\title{
The spectrum of Evans' syndrome
}

\author{
Süreyya Savaşan, Indira Warrier,Yaddanapudi Ravindranath
}

\begin{abstract}
Eleven patients (10 boys, one girl) with Evans' syndrome with a median follow up time of 8.0 years were evaluated retrospectively. Six patients had either persistent hepatosplenomegaly or generalised lymphadenopathy, or both. In five patients, an increase in lymph node and/or spleen size was observed during the exacerbations of cytopenias. Seven patients had quantitative serum immunoglobulin abnormalities at the time of presentation. There were associated systemic manifestations in nine patients. Various forms of treatment were used with mixed results. Four patients died from sepsis and haemorrhage; four had complete recovery-two after splenectomy. These findings show that Evans' syndrome is a heterogeneous disorder with significant morbidity and mortality. High incidence of quantitative serum immunoglobulin abnormalities, lymphoid hyperplasia, and associated systemic manifestations suggest that Evans' syndrome may represent a stage of a more broad spectrum, generalised immune dysregulation.

(Arch Dis Child 1997;77:245-248)
\end{abstract}

Keywords: Evans' syndrome; lymphoproliferation; serum immunoglobulins; immune dysregulation

Evans' syndrome is characterised by simultaneous or sequential development of autoimmune thrombocytopenia (AITP) and autoimmune haemolytic anaemia (AIHA). ${ }^{1}$ A significant number of patients also have neutropenia. Despite therapeutic interventions, the great majority of patients with Evans' syndrome have a chronic and relapsing course, which is associated with significant morbidity and mortality. ${ }^{2}$

Autoantibodies against erythrocytes, platelets, and neutrophils have been shown in Evans' syndrome. Both the occurrence of isolated episodes of thrombocytopenia and haemolytic anaemia and the results of in vitro studies have suggested the role of non-cross reacting autoantibodies targeted at different antigenic determinants on red cells and platelets. ${ }^{3}{ }^{4}$ A pioneering report by Zuelzer et al suggested a generalised immune dysregulation in some patients with AIHA who had a more protracted and chronic course. ${ }^{5}$ These patients were characterised by generalised lymphadenopathy, abnormal serum immunoglobulins, and idiopathic thrombocytopenic purpura (ITP) as well as AIHA (in almost half of the cases). Both humoral and cellular immune response abnormalities have been shown in Evans' syndrome. ${ }^{46}$ AITP and AIHA have been found to be associated with primary immunodeficiencies, ${ }^{7-12}$ collagen vascular diseases, lymphoproliferative disorders, and recently, autoimmune lymphoproliferative syndrome (ALPS). ${ }^{13-16}$ All of these findings prompted us to review our cases of Evans' syndrome in order to see the spectrum of this disorder in children.

\section{Patients and methods}

Inpatient and outpatient charts of 16 patients with AITP and AIHA followed up by the haematology/oncology department at the Children's Hospital of Michigan in the past 20 years were reviewed. Five patients were excluded from the study; two because of very short follow up time (less than a year), two with systemic lupus erythematosus (SLE), and one with HIV infection.

\section{Results}

Eleven patients were included in the study. Median age at presentation was 5.5 years $(0.7-$ 14.2), median follow up time 8.0 years (2.3-11.8). There was a male preponderance in our series (10 boys and one girl). Six were African-Americans $(55 \%)$, four white $(36 \%)$, and one Hispanic (9\%).

All patients presented with thrombocytopenia and bleeding symptoms. Only two had haemolytic anaemia at presentation. There was neutropenia in five of the patients initially, and another patient developed it later (55\%). Nine patients $(82 \%)$ had positive direct Coombs test at presentation before any treatment, while two became positive later. Six patients $(55 \%)$ showed clinical signs of lymphoid hyperactivity; two had hepatosplenomegaly and generalised lymphadenopathy, one had isolated splenomegaly, and three had generalised lymphadenopathy only. An increase in lymph node and/or spleen size was observed during the exacerbations of the cytopenias in five patients.

Nine patients $(82 \%)$ had other systemic manifestations or disorders. Lymphoid hyperplasia was confirmed by microscopic evaluation of lymph nodes or spleen tissue in four patients. Three were found to have portal triaditis (two of the three at necropsy-one patient died of postsplenectomy sepsis, the other from intracranial bleeding). Three patients were diagnosed with interstitial nephritis (two at necropsy). Transient low titre antinuclear antibody positivity was seen in two, eczema in two, insulin dependent diabetes mellitus, cerebral infarct (lupus anticoagulant negative), Guillain-Barré syndrome, and migraine in one each. 
Table 1 Frequency of some of the physical and laboratory findings

\begin{tabular}{lc}
\hline Finding & No (\%) \\
\hline Presentation & $11(100)$ \\
Thrombocytopenia & $2(18)$ \\
Haemolytic anaemia & $5(45)$ \\
Neutropenia & $7(64)$ \\
Quantitative serum immunoglobulin & $9(82)$ \\
$\quad$ abnormalities & $6(55)$ \\
Positive direct Coombs test & $6(55)$ \\
$\begin{array}{l}\text { Follow up } \\
\text { Neutropenia }\end{array}$ & $9(82)$ \\
$\quad \begin{array}{l}\text { Hepatomegaly, splenomegaly, and/or } \\
\text { lymphadenopathy }\end{array}$ & \\
$\quad \begin{array}{l}\text { Associated systemic manifestations or } \\
\text { disorders }\end{array}$ & \\
\hline
\end{tabular}

Quantitative serum immunoglobulin abnormalities were observed at the time of presentation, before any treatment and during follow up. Initially seven patients $(64 \%)$ had abnormal levels: one panhypogammaglobulinaemia, one panhypergammaglobulinaemia, one low IgA, one high IgG, one high IgA, and two high IgE. Three others developed abnormal concentrations during follow up and different abnormalities had emerged with the disappearance of the previous abnormalities in two. Table 1 summarises these results.

Nine patients had at least one episode of simultaneous thrombocytopenia and haemolytic anaemia. Otherwise, types of episodes varied among patients and also for a given individual. Various forms of treatment such as steroids, intravenous immunoglobulin G, immunosuppressive drugs (cyclosporin, cyclophosphamide, azathioprine), and splenectomy were used either alone or in combination with variable response. Four patients recovered $(36 \%)$, including two of the five patients who had undergone splenectomy. Four patients died $(36 \%)$, two from sepsis after splenectomy and two from intracranial bleeding (necropsy results available in one). Three patients are currently on treatment with stable counts.

\section{Discussion}

Although approximately $20-25 \%$ of children with AIHA develop immune thrombocytopenia, evolution into Evans' syndrome is relatively uncommon in patients with ITP. ${ }^{2517}$ However, in our series, all patients presented with thrombocytopenia; only two of them had clinically evident autoimmune haemolysis at initial presentation. This emphasises the significance of obtaining a Coombs test in all patients newly diagnosed with ITP and during their follow up. Frequency of neutropenia in our series is consistent with the current literature. ${ }^{18}$ Although neutropenia was more resistant to various forms of treatment used, these patients did not develop life threatening infections, but had experienced recurrent respiratory tract infections (for example, sinusitis, otitis, bronchitis).

Another striking finding in our study was the high frequency of quantitative serum immunoglobulin abnormalities, although the significance of the increased $\operatorname{IgE}$ in two patients is difficult to evaluate. Observed changes in serum immunoglobulin concentrations could be attributed in part to the treatments used; however, initial abnormality may be an element of a more generalised immune dysregulation disorder.

The presence of hepatosplenomegaly or generalised lymphadenopathy, or both, and the demonstration of lymphoid hyperplasia by microscopic evaluation of tissue specimens, suggested a link between the development of autoimmune cytopenias and lymphoid hyperplasia. This was further supported by the observation of an increase in lymph node and/or spleen size during the exacerbations of the cytopenias in some patients. These findings, and the presence of other systemic manifestations or disorders, such as interstitial nephritis, Guillain-Barré syndrome, insulin dependent diabetes mellitus, portal triaditis, asthma, and eczema are indicative of a more generalised immune dysregulation in Evans' syndrome. The clinical course, in most of the patients with Evans' syndrome, is reminiscent of patients with chronic ITP or chronic AIHA. Our findings are similar to the AIHA group III patients with massive lymphadenopathy and serum immunoglobulin abnormalities, some with additional thrombocytopenia and neutropenia reported by Zuelzer et al. ${ }^{5}$

It has been known that AITP and AIHA may be the early features of evolving SLE. Miescher et al described a group of patients, mainly young adults with AIHA or AITP, or both, who had laboratory findings of positive low titre antinuclear antibodies and very low antidsDNA antibody level without a clear cut diagnosis of SLE. ${ }^{19}$ The authors postulated that these cases represented certain transitional forms of autoimmune disorders similar to those seen in New Zealand black mice with AIHA and/or AITP. Several studies on New Zealand black and New Zealand white mice have implicated the requirement of multiple genetic defects for the development of a full blown disease.

Fas antigen, also known as APO-1, is expressed on activated $T$ cells and $B$ cells and plays an important part in the regulation of the immune response by inducing apoptosis upon binding with its ligand (FasL). It has been shown that mice with the lymphoproliferation $(l p r)$ mutations develop generalised lymphadenopathy and a SLE-like disease and have defects in the Fas antigen gene. ${ }^{20}$ The mice with generalised lymphoproliferation disorder (gld) also develop a clinicopathological condition similar to mice with $l p r$ mutation and carry FasL gene mutations. ${ }^{21}$ FasL gene mutation has been shown in a patient with SLE and lymphoproliferative disease. ${ }^{22} \mathrm{~A}$ novel disorder, ALPS, characterised by generalised lymphoproliferation, hypergammaglobulinaemia and multiple autoimmune phenomena, including AIHA and AITP, has been described in humans, and this disorder was later shown to be associated with Fas gene defects. ${ }^{13-16}$

Development of AIHA and AITP is not infrequent in patients with selective IgA deficiency or common variable immuno- 
deficiency. ${ }^{9-12}$ It has been shown that some patients with selective IgA deficiency may evolve into common variable immunodeficiency disease (CVID). ${ }^{9}{ }^{23}$ Twenty per cent of patients with CVID develop some type of autoimmunity, and immune cytopenia is the leading form. ${ }^{10}$ Lymphoproliferative lesions are not uncommon in CVID. ${ }^{24}{ }^{25}$ It has been shown that B cells from a subset of patients with CVID have increased Fas antigen expression and undergo enhanced apoptosis. ${ }^{26} 27$

Some patients with Evans' syndrome and panhypogammaglobulinaemia are reminiscent of those with CVID, whereas another subgroup of patients with Evans' syndrome and hypergammaglobulinaemia resembles ALPS. It is obvious that there are several common clinical and laboratory features in SLE, ALPS, CVID, and Evans' syndrome and these disorders may overlap. AIHA, AITP, generalised lymphoproliferation, and quantitative serum immunoglobulin abnormalities (either low or high concentrations) are common in all these disorders. Fas-FasL system defects (either decreased or increased activity) are seen in SLE, ALPS, and CVID, but have not been studied in Evans' syndrome. It may be useful to study Fas-FasL system in Evans' syndrome. Some of the cases of immune dysregulation may represent variable expression of a complex and continuous spectrum and it may be difficult to clearly define the boundaries between these various disorders. Autoimmune cytopenias and lymphoproliferation, as a result of defective control of the normal immune response in a patient with an underlying immunodeficiency, can be proposed as one of the pathophysiological mechanisms in Evans' syndrome.

The clinical course is complicated and outcome is poor in patients with Evans' syndrome when compared with patients with isolated ITP or AIHA. No consistent response was seen with the various forms of treatment used. Although successful results have been reported with various treatments occasionally, ${ }^{28-32}$ many patients became dependent or refractory to the treatment in our series. Permanent complete recovery after splenectomy is uncommon in Evans' syndrome in contrast with chronic ITP or AIHA, which can explain the low complete recovery rate in our splenectomised patients. ${ }^{17}{ }^{33}$ The high mortality rate reported here is similar to that reported from the other centres. ${ }^{18}$ The findings from our retrospective analysis suggest that Evans' syndrome in children is a heterogeneous and serious disorder with significant morbidity and mortality, and the current therapeutic modalities are more palliative than curative.

In conclusion, some cases of Evans' syndrome, especially those with a more protracted course, may belong to a group of disorders characterised by the triad of immune deficiency, autoimmunity, and lymphoproliferation. The treatment of these patients is complicated and remains a challenge. Studies to evaluate the involvement of Fas-FasL system may shed new insights into pathogenesis and possibly development of new therapeutic approaches.

This work was supported by the Georgie Ginopolis Fund.

1 Evans RS, Takahashi K, Duane RT, Payne R, Lie CK. Primary thrombocytopenic purpura and acquired hemolytic anemia: evidence for common etiology. Arch Intern Med 1951;87:48-65.

2 Pui C-H, Wilimas J, Wang W. Evans' syndrome in childhood. F Pediatr 1980;97:754-8.

3 Pegels JG, Helmerhorst FM, van Leeuwen EF, van de Plasvan Dalen C, Engelfriet CP, von dem Borne AEGKR. The Evans' syndrome: characterisation of the responsible utoantibodies. Br 7 Haematol 1982;51:445-50.

4 Miller BA, Beardsley DS. Autoimmune pancytopenia of childhood associated with multisystem disease manifestations. F Pediatr 1983;103:877-81.

5 Zuelzer WW, Mastrangelo R, Stulberg CS, Poulik MD, Page $\mathrm{RH}$, Thompson RI. Autoimmune hemolytic anemia. Natural history and viral-immunologic interactions in childhood. Am f Med 1970;49:80-93.

6 Wang W, Herrod H, Pui C-H, Presbury, G, Wilimas J. Immunoregulatory abnormalities in Evans' syndrome. Am 7 Hematol 1983;15:381-90.

7 Ashby GH, Evans DIK. Cartilage hair hypoplasia with thrombocytopenic purpura, autoimmune haemolytic anaemia and cell-mediated immunodeficiency. $7 \mathrm{R}$ Soc Med mia and cell-med.

8 Villiger PM, Fey MF, Lotz M, Tobler A. IgG ${ }_{2}$ and IgG $_{4}$ subclass deficiency and Evans' syndrome in an adult patient. $\mathcal{F}$ Allergy Clin Immunol 1992;90:693-4.

9 Sanal Ö, Ersoy F, Metin A, Tezcan I, Berkel AI, Yel L. Selective IgA deficiency with unusual features: development of common variable immunodeficiency, Sjögren's syndrome, autoimmune hemolytic anemia and immune thrombocytopenic purpura. Acta Pediatr fpn 1995;37:5269.

10 Hansen OP, Srrensen CH, Astrup L. Evans' syndrome in IgA deficiency. Episodic autoimmune haemolytic anaemia and thrombocytopenia during a 10 years observation period. Scand F Haematol 1982;29:265-70.

11 Cuesta B, Fernãndez J, Pardo J, Paramo JA, Gomez C, Rocha E. Evans' syndrome, chronic active hepatitis and focal glomerulonephritis in IgA deficiency. Acta Haematol 1986;75:1-5.

12 Sneller MC, Strober W, Einstein E, Jaffe JS, CunninghamRundles C. New insights into common variable immunodeficiency. Ann Intern Med 1993;118:720-30.

13 Sneller MC, Straus SE, Jaffe ES, et al. A novel lymphoproliferative/autoimmune syndrome resembling murine lpr/gld disease. $\mathcal{F}$ Clin Invest 1992;90:334-41.

14 Fisher GH, Rosenberg FJ, Straus SE, et al. Dominant interfering Fas gene mutations impair apoptosis in a human autoimmune lymphoproliferative syndrome. Cell 1995;81: 935-46.

15 Rieux-Laucat F, Le Deist F, Hivroz C, et al. Mutations in Fas associated with human lymphoproliferative syndrome and autoimmunity. Science 1995;268:1347-9.

16 Le Deist F, Emile J-F, Rieux-Laucat F, et al. Clinical, immunological, and pathological consequences of Fas-deficient conditions. Lancet 1996;348:719-23.

17 Habibi B, Homberg JC, Schaison G, Salmon C. Autoimmune hemolytic anemia in children. A review of 80 cases. Am $\mathcal{F}$ Med 1974;56:61-9.

18 Wang WC. Evans' syndrome in childhood: pathophysiology, clinical course, and treatment. Am f Pediatr Hematol Oncol 1988;10:330-8.

19 Miescher PA, Tucci A, Beris P, Favre H. Autoimmune hemolytic anemia and/or thrombocytopenia associated with lupus parameters. Semin Hematol 1992;29:13-7.

20 Watnabe-Fukunaga R, Brannan CI, Copeland NG, Jenkins NA, Nagata S. Lymphoproliferation disorder in mice explained by defects in Fas antigen that mediates apoptosis. Nature 1992;356:314-7.

21 Mountz JD, Zhou T, Su X, Wu J, Cheng J. The role of programmed cell death as an emerging new concept for the pathogenesis of autoimmune diseases. Clin Immunol Immupathogenesis of autoimmune disease $1996 ; 80$ (suppl):S2-14.

22 Wu J, Wilson J, He J, Xiang L, Schur PH, Mountz JD. Fas ligand mutation in a patient with systemic lupus erythematosus and lymphoproliferative disease. $\mathcal{F}$ Clin Invest 1996;98:1107-13

23 Espanol T, Catala M, Hernandez M, Caragol I, Bertran M. Development of a common variable immunodeficiency in IgA-deficient patients. Clin Immunol Immunopathol 1996; 80:333-5.

24 Curtin JJ, Murray JG, Apthorp LA, Franz AM, Webster DB. Mediastinal lymph node enlargement and splenomegaly in primary hypogammaglobulinemia. Clin Radiol 1995;50:

Sander CA, Medeiros LJ, Weiss LM, Yano T, Sneller MC, Jaffe ES. Lymphoproliferative lesions in patients with common variable immunodeficiency syndrome. Am $\mathcal{f}$ Pediatr Hematol Oncol 1992;16:1170-82.

26 Saxon A, Keld B, Diaz-Sanchez D, Guo B-C, Sidell N. B cells from a distinct subset of patients withcommon variable immunodeficiency (CVID) have increased 
CD95 (Apo-1/fas), diminished CD38 expression, and CD95 (Apo-1/fas), diminished CD38 expression, and undergo

27 Guo B-C, Saxon A. B cell lines from a subset of patients with common variable immunodeficiency undergo enhanced apoptosis associated with an increased display of CD95 Apo-1/fas), diminished C 38 expression, and decreased IgG and IgA production. Cell Immunol 1995;166:83-92.

28 Oda H, Honda A, Sugita K, Nakamura A, Nakajima H. High-dose intravenous intact IgG infusion in refractory autoimmune hemolytic anemia (Evans' syndrome). F Pediatr 1985;107:744-6.

29 Nuss R, Wang W. Intravenous gamma globulin for thrombocytopenia in children with Evans' syndrome. $A m \mathcal{F}$ Pediatr Hematol Oncol 1987;9:164-7.
30 Petrides PE, Hiller E. Autoimmune hemolytic anemia combined with idiopathic thrombocytopenia (Evans' syndrome). Sustained remission in a patient following high-dose intravenous gamma-globulin therapy. Clin Invest Med 1992;70:38-9.

31 Scaradavou A, Bussel J. Treatment of refractory Evans' syndrome with alternate-day cyclosporine and prednisone. $\mathcal{F}$ Pediatr Hematol Oncol 1995;17:290-5.

32 Rackoff WR, Manno CS. Treatment of refractory Evans' syndrome with alternate-day cyclosporine and prednisone. Am F Pediatr Hematol Oncol 1994;16:156-8.

33 Simons SM, Main CA, Yaish HM, Rutzky J. Idiopathic thrombocytopenic purpura in children. F Pediatr 1975;87: $16-22$. 